The Astrophysical Journal, 548:L95-L98, 2001 February 10

(c) 2001. The American Astronomical Society. All rights reserved. Printed in U.S.A.

\title{
RAPID SUBMILLIMETER BRIGHTENINGS ASSOCIATED WITH A LARGE SOLAR FLARE
}

\author{
P. Kaufmann, J.-P. Raulin, E. Correia, J. E. R. Costa, C. G. Giménez de Castro, and A. V. R. Silva \\ CRAAM/CRAAE, ${ }^{1}$ Universidade Presbiteriana Mackenzie, Rua da Consolação 896, São Paulo, SP-013202-907, Brazil \\ H. LeVATO \\ Complejo Astronómico El Leoncito, Casilla de Correo 467, Av. España 1512 Sur, San Juan 5400, Argentina \\ M. Rovira, C. Mandrini, and R. Fernández-Borda \\ Instituto de Astronomia y Física del Espacio, Casilla de Correo 67, Sucursal 28, Buenos Aires 1428, Argentina \\ AND \\ O. H. BAUER \\ Max-Planck-Institut für extraterrestrische Physik, Giessenbachstrasse Postfach 1603, Garching, D-85740, Germany \\ Received 2000 October 3; accepted 2000 December 6; published 2001 February 12
}

\begin{abstract}
We present high time resolution observations of Active Region 8910 obtained simultaneously at 212 and $405 \mathrm{GHz}$ during a large $\mathrm{H} \alpha$ flare, which produced a soft X-ray class X1.1 event. Data were obtained with the new solar submillimeter telescope recently installed at the El Leoncito Observatory to explore this poorly known part of the solar emission spectrum. A small slow submillimeter enhancement ( $\leq 300 \mathrm{sfu})$ was associated to bulk emissions at X-rays, $\mathrm{H} \alpha$, and microwaves. The event exhibited numerous submillimeter-wave $100-300 \mathrm{~ms}$ duration spikes, the larger ones with fluxes on the order of 220 and $500 \mathrm{sfu}( \pm 20 \%)$ at 212 and $405 \mathrm{GHz}$, respectively. A dramatic increase in the incidence rate of submillimeter spikes sets in as a new large loop system appears in AR 8910, and X-ray emission increases nearly $1 \mathrm{hr}$ before the large flare. The brightening incidence rate ( 20 per minute) correlates well with the large flare light curves at X-rays and $\mathrm{H} \alpha$. The submillimeter spikes may be associated to microflares, waves, or quakes in flaring active regions.
\end{abstract}

Subject headings: Sun: flares — Sun: radio radiation

\section{INTRODUCTION}

There is a need to know solar flare emission as well as active region activity regimes, at frequencies larger than $100 \mathrm{GHz}$ for critical tests, to understand particle acceleration and energyloss mechanisms in the magnetoactive plasmas (Stein \& Ney 1963; Shklovsky 1964; Beckman 1968; Kaufmann et al. 1986; McClements \& Brown 1986; Klein 1987). There are numerous examples of burst spectra in the microwave-millimeter wave range with intensities increasing toward the highest frequencies for which observations were available $(\leq 100 \mathrm{GHz})$, suggesting the existence of an emission component with maximum intensity somewhere in the submillimeter-IR range (Croom 1973; Kaufmann et al. 1985; Kaufmann 1996 and references therein). However, flare observations are poorly known along nearly three decades of submillimeter-IR frequencies. The only known attempts to observe flares at submillimeter waves made use of cooled bolometers in optical telescopes, more than 30 years ago, producing a few suggestive, although not fully conclusive, results. Clark \& Park (1968) found brightness variations on the order of $100 \mathrm{~K}$ on solar active regions at about $250 \mathrm{GHz}$, with time resolution limited to 1 minute, without any other flaring counterpart reported. Hudson (1975) reported $10-50 \mathrm{~K}$ fluctuations at $850 \mathrm{GHz}(1 \mathrm{~m}$ time resolution) and at $12 \mathrm{THz}$ ( $1 \mathrm{~s}$ time resolution).

A new solar submillimeter-wave telescope (SST; Kaufmann et al. 2000) was recently installed at the El Leoncito Astronomical Complex, CASLEO, in the Argentinean Andes. It has a radome-enclosed $1.5 \mathrm{~m}$ reflector and can operate simultaneously with four total-power heterodyne room temperature receivers at $212 \mathrm{GHz}$ and two at $405 \mathrm{GHz}$, with $1 \mathrm{~ms}$ time resolution. While SST was undergoing final alignment adjust-

\footnotetext{
${ }^{1}$ CRAAE-Centro de Rádio Astronomia e Aplicações Espaciais is a joint center between Mackenzie, INPE, USP, and UNICAMP.
}

ments, a number of short campaigns of solar observations were carried out. The provisional aperture efficiencies in 2000 March, derived from Jupiter observations, were still small $(2.5 \%$ at $405 \mathrm{GHz}$ and $5 \%$ at $212 \mathrm{GHz}$, with uncertainties of $\pm 20 \%$ ), with main-beam mean angular sizes larger than the nominally expected, as shown in Figure 1. The pointing accuracy was better than $1^{\prime}$, and tracking accuracy was on the order of 3".6.

\section{FLARE OBSERVATIONS AT SUBMILLIMETER WAVES}

The SST was tracking the NOAA Active Region 8910 when a large flare occurred on 2000 March 22, producing a class X1.1 GOES soft X-ray event at about 1830-1930 UT. Microwaves were reported for this event by the Radio Solar Telescope Network (Sol.-Geophys. Data 2000), and it was also observed by the $7 \mathrm{GHz}$ solar polarimeter at Itapetinga Radio Observatory in Brazil. Multiple microwave frequency observations were obtained by the Owens Valley Solar Array (Gallagher, Gary, \& Lee 2000).

UV images from the Solar and Heliospheric Observatory (SOHO)/EUV Imaging Telescope (EIT) and Transition Region and Coronal Explorer (TRACE) space experiments were made available (S. L. Freeland 2000, private communication). $\mathrm{H} \alpha$ observations were obtained by the $\mathrm{H} \alpha$ Solar Telescope for Argentina (HASTA; Bagalá et al. 1999), also located at the El Leoncito area in the neighboring OAFA observatory.

\subsection{The Slow-Emission Time Profiles}

Figure 1 displays the SST beams with respect to the solar disk, with beam $5(405 \mathrm{GHz})$ tracking AR 8910. Figure 2 shows the emissions in a compressed timescale from 1710-2010 UT. At the top we show the relative variations in channel 4, $212 \mathrm{GHz}$, in units of antenna temperature, corrected for at- 


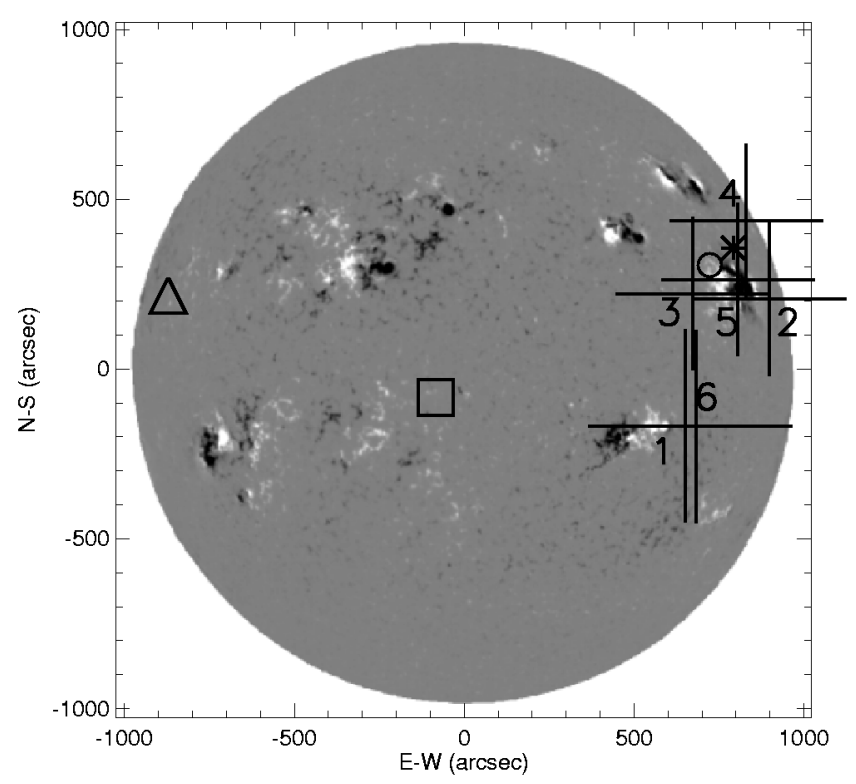

FIG. 1.-SST six beams (1-4 at $212 \mathrm{GHz}, 5-6$ at $405 \mathrm{GHz})$ displayed on a KPNO magnetogram for 2000 March 22, showing beam 5 pointing at AR 8910 at about the time the event. Bars indicate the approximate beam half-power widths at the time of these observations. Approximate position for the bright spike shown in Fig. $3 a$ is indicated by an asterisk, and the circle indicates an estimated position for the small slow submillimeter-wave enhancement (Fig. 2, top). The square and the triangle indicate beam 5 tracking the quiet Sun on the disk and on the limb, respectively, with $5 \mathrm{~s}$ samples shown in Figs. $3 b$ and $3 c$.

mospheric transmission. Below is the GOES-8 0.5-4 $\AA$ time profile together with the $7 \mathrm{GHz}$ total flux (in sfu; $1 \mathrm{sfu}=$ $10^{-22} \mathrm{~W} \mathrm{~m}^{-2} \mathrm{~Hz}^{-1}$ ). The bottom plot is the total $\mathrm{H} \alpha$ light curve obtained by HASTA, in relative units, adding emissions from AR 8910 and from the nearby AR 8917, located northeast of AR 8910, still within the field of view of SST beams. Labels $\mathrm{A}$ and $\mathrm{B}$ in Figure 2 indicate $5 \mathrm{~s}$ samples shown in Figure 3.

The small slow submillimeter-wave antenna temperature enhancement observed with beam 4 ( $212 \mathrm{GHz}$; see Fig. 2, top $)$ was also observed in beams 2 and 3 but not in beam 1. Using the multiple-beam technique for source position determination (Giménez de Castro et al. 1999 and references therein), it was possible to derive a very approximate position, indicated by a circle in Figure 1, with a corrected antenna temperature of $\leq 100 \mathrm{~K}$ at $212 \mathrm{GHz}$. Similar enhancements at $405 \mathrm{GHz}$ could not be identified because of the much larger long-term atmospheric propagation fluctuations.

\subsection{Fast Brightenings at Submillimeter Waves Only}

In Figure 3, the arrows show examples of two typical submillimeter-wave brightenings as detected by the SST beams when observing the active center at time A in Figure 2 compared to the submillimeter-wave emissions observed when tracking a quiet-Sun region (Fig. 1, square) at time B and when tracking the quiet east limb (Fig. 1, triangle) the following day. On the active region, we observed a spiky pattern with numerous larger brightenings, well-detected by the beams closer to the flaring region (beams 2, 3, and 4 at $212 \mathrm{GHz}$ and beam 5 at $405 \mathrm{GHz}$ ) and nearly undetected by the distant beams (beam 1 at $212 \mathrm{GHz}$ and beam 6 at $405 \mathrm{GHz}$ ).

The correlation of antenna temperatures for beams 2,3 , and 4 (at $212 \mathrm{GHz}$ ) for the largest spikes shown in Figure $3 a$ is consistent to a single burst position, shown by an asterisk in

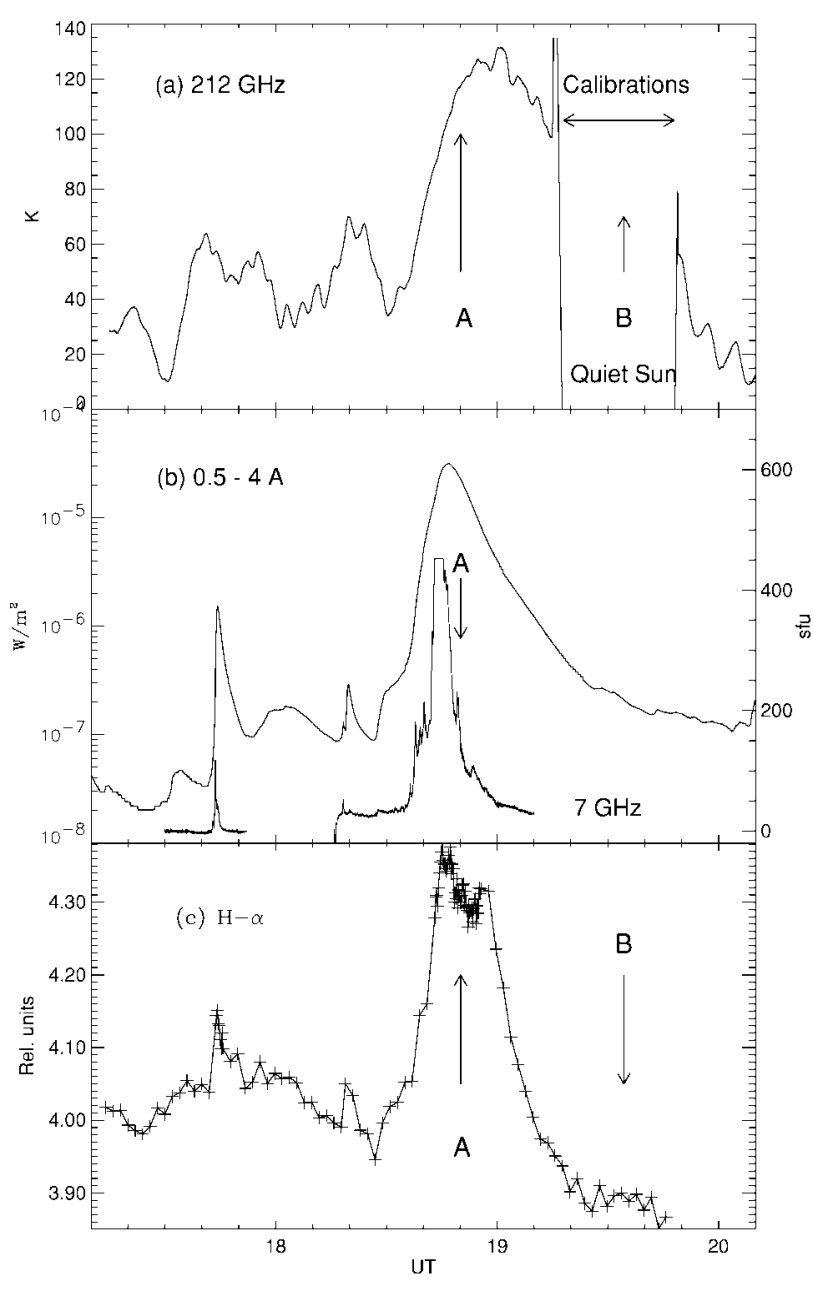

FIG. 2. - 2000 March 22 flare time profiles in compressed timescale at different wavelengths.

Figure 1. The largest spike shown in Figure $3 a$ has peak antenna temperatures, corrected for atmospheric transmission, of about $60 \mathrm{~K}$ in both beam $4(212 \mathrm{GHz})$ and $5(405 \mathrm{GHz})$, which can be corrected for the values at the source position indicated by the asterisk in Figure 1, assuming approximate Gaussian beams, and the same location at both frequencies. We estimate a final corrected antenna temperature at the burst position of about $80 \mathrm{~K}$ at $405 \mathrm{GHz}$ and $70 \mathrm{~K}$ at $212 \mathrm{GHz}$. A qualitative inspection shows that various of these larger spikes along the whole event have similar characteristics, with half-power duration in the range of $100-300 \mathrm{~ms}$.

Limb-tracking effects could not have produced the larger spikes shown in Figure $3 a$. To demonstrate this, and to avoid fewer solar spikes present in AR 8910 before and after the main event, we show an example of quiet-Sun limb-tracking on March 23 in Figure 3c, with the beam 5 location relative to the limb similar to that during the flare. The observed modulation pattern, with frequency varying with elevation/azimuth antenna velocities and time, is caused by tracking corrections of 3".6, corresponding to encoder reading accuracy, monitored permanently. We see that the modulation is typical and its amplitude does not exceed $15 \mathrm{~K}$ in channel 4, in agreement with computations for a beam partially filled by the solar disk. Moreover, the counting of limb-tracking time structures led to zero counts for temperature variations above $17 \mathrm{~K}$. Alternatively, if a steady, emitting point source is placed within the 


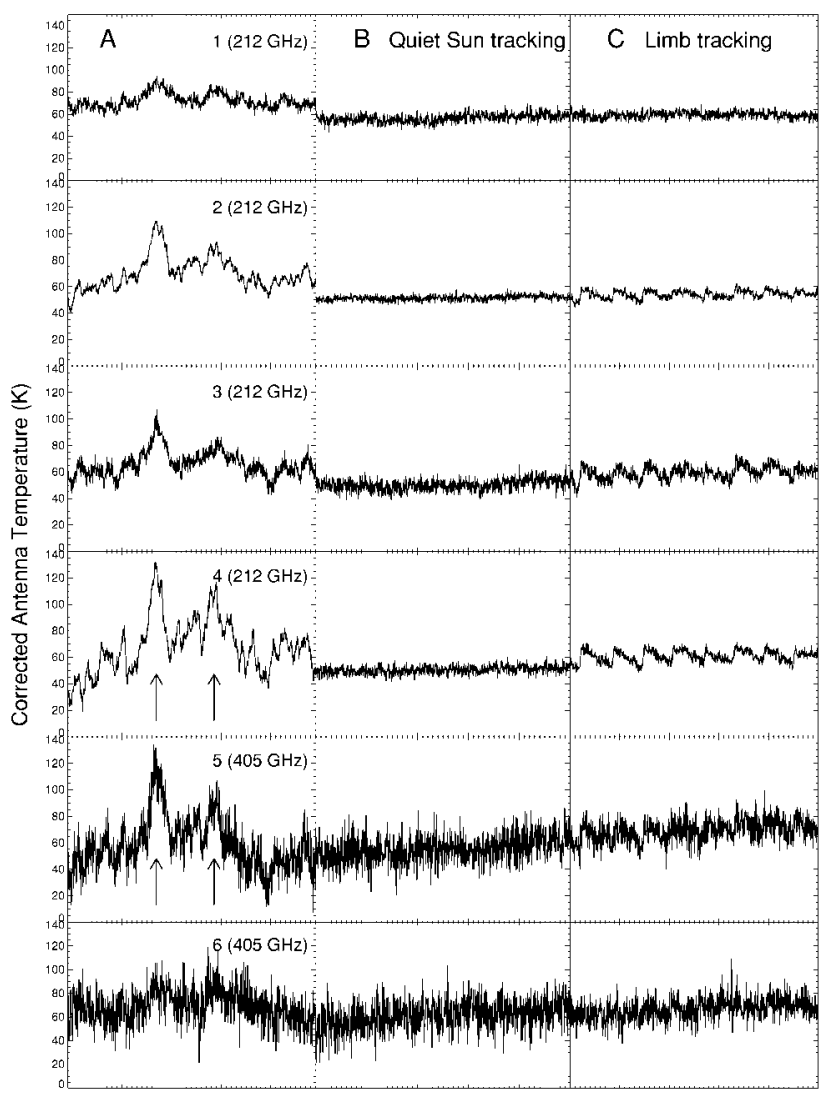

FIG. 3.-The $5 \mathrm{~s}, 5 \mathrm{~ms}$ resolution samples showing (a) two typical brightenings (arrows), well defined at 212 and $405 \mathrm{GHz}$ by the beams closer to the burst source $(2,3,4$, and 5$),(b)$ emission when tracking a solar quiet region (S05 E05; Fig. 1, square), and (c) emission when tracking the quiet solar limb (N13 E68; Fig. 1, triangle) on March 23, 1540 UT.

beams, the temperature fluctuations due to such tracking variations should be anticorrelated for at least two beams, which was not observed. Moreover, a $100 \mathrm{~K}$ steady point source would fluctuate by less than $1 \mathrm{~K}$, which is undetectable.

Antenna temperatures were corrected for zenith opacity of 1.2 nepers at $405 \mathrm{GHz}$ and 0.15 nepers at $212 \mathrm{GHz}$ as a function of elevation angle. It is known that at millimeter waves, variations may occur in clear sky conditions in rare occasions (Altenhoff et al. 1987; Makhmutov et al. 1998), on timescales of several to tens of seconds, caused by inhomogeneities on the order of or larger than $300 \mathrm{~m}$ at tropospheric heights of 5-10 km, corresponding to angular sizes considerably larger than the solar disk. Therefore, antenna temperature variations should be similar for all SST beams on the solar disk, which was not observed. Moreover, if the observed subsecond brightenings were produced by atmospheric inhomogeneities, this would imply unrealistically small or fast traveling cells. Besides, one should expect to observe many large "negative atmospheric" spikes as well, which were not found.

The burst flux densities for the submillimeter-wave typical spike emission (the largest shown in Fig. $3 a$ ) were estimated using provisional antenna aperture efficiencies. We obtained $500 \mathrm{sfu}$ at $405 \mathrm{GHz}$ and $220 \mathrm{sfu}$ at $212 \mathrm{GHz}$ with uncertainties of about $\pm 20 \%$. The positive spectral index for flux $\alpha(S \propto$ $\nu^{\alpha}$, where $S$ is the flux density and $\nu$ is the frequency) lies somewhere between $\alpha=0.6$ and 1.9 for the extreme values in the uncertainties in flux determinations. This result can be verified by an independent estimate obtained from the ratio of

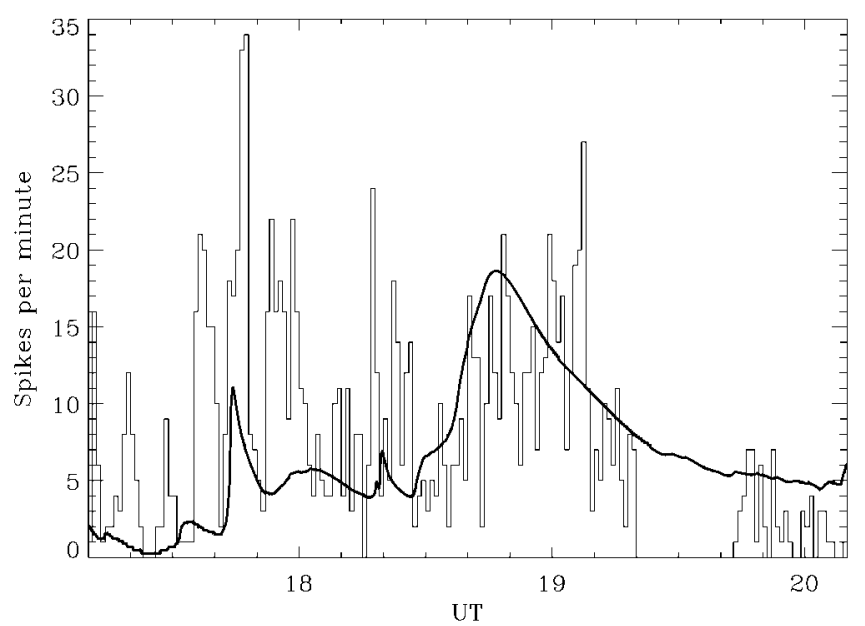

Fig. 4.-Incidence time rate of brighter spikes $(\geq 25 \mathrm{~K}$ in beam $4,212 \mathrm{GHz})$ per minute, superposed on the GOES X-ray time profile.

observed corrected antenna temperatures, $T_{1}$ and $T_{2}$, assuming that the burst sources have about the same angular size at both frequencies, $\nu_{1}(405 \mathrm{GHz})$ and $\nu_{2}(212 \mathrm{GHz}): \alpha \sim$ $\ln \left[\left(T_{1} / T_{2}\right)\left(\nu_{1} / \nu_{2}\right)^{2}\right] / \ln \left(\nu_{1} / \nu_{2}\right)$. Using $T_{1} \sim 80 \mathrm{~K} \pm 10 \%$ and $T_{2} \sim 70 \mathrm{~K} \pm 10 \%$ gives a positive spectral index somewhere in the range $\alpha=1.9-2.5$.

\section{INCIDENCE RATE OF BRIGHTER SUBMILLIMETER-WAVE SPIKES}

In Figure 4, we show the brighter spikes' incidence rate detected with channel $4(212 \mathrm{GHz})$, together with the GOES $\mathrm{X}$-ray time profile for comparison. Rise and fall pulses larger than a $25 \mathrm{~K}$ threshold were counted using a peak-finding algorithm (Raulin et al. 1998) applied on $60 \mathrm{~ms}$ integrated data such that all brighter structures were included. We found that the accidental superposition of smaller and faster structures on the tracking ripple was negligible by comparing data recorded simultaneously with antenna encoder readings.

Approximately 1300 spikes were counted. We made an independent count on channel $5(405 \mathrm{GHz})$ for spikes brighter than $40 \mathrm{~K}$ (more than twice the limb-tracking ripple) and confirm the same result shown in Figure 4. It can be seen that the incidence of brighter spikes increases dramatically at about $1730 \mathrm{UT}$, as the X-ray emission increases, attaining rates of about 20-25 per minute. The main maximum correlates well to the flare bulk emission time profile at X-rays as well as at $\mathrm{H} \alpha$ and microwaves (1830-1930 UT). Other brightening concentrations were found at 1730-1800 and 1810-1830 UT. They may have some relationship to two $\mathrm{H} \alpha$ flares detected by HASTA on AR 8917 before the large event. However, it is not evident to which active region the X-ray and microwave emissions are coming from at about 1745,1800 , and 1820 UT, since they were observed by instruments with fields of view larger than the Sun. Indeed, by inspecting UV TRACE and SOHO/ EIT movies centered on AR 8910 (S. L. Freeland 2000, private communication), we see that important activity occurs from about 1700 UT. TRACE has shown a major change in the magnetic topology of AR 8910, with the appearance of a new large active loop system somewhere between the frames taken at 1715 and $1740 \mathrm{UT}$, remaining active until at least $1850 \mathrm{UT}$. There were no other optical observations with subsecond cadence rates for this event. 


\section{DISCUSSION AND CONCLUDING REMARKS}

The slow enhancement observed at $212 \mathrm{GHz}$ together with the class X flare corresponds to an approximate flux density of $\leq 300 \mathrm{sfu}$ (assuming the source is small compared to the antenna beams), which might be attributed to the thermal component of the bulk emission observed at microwaves extended to the submillimeter waves. On the other hand, the temperature of the X-ray-emitting plasma was $2 \times 10^{7} \mathrm{~K}$, which was inferred from the ratio between the two GOES soft X-ray bands. If both the submillimeter-wave and X-ray sources were the same, from the ratio between the source and the submillimeterwave beam solid angles, the small slow $212 \mathrm{GHz}$ temperature enhancement $(\leq 100 \mathrm{~K})$ should imply an unlikely submillimeterwave source size less than $1^{\prime \prime}$, which might be an indication that it is optically thick, deeply immersed in a dense plasma. Indeed, for thermal emissions, a spectral turnover frequency above $405 \mathrm{GHz}$ implies densities of about $10^{15} \mathrm{~cm}^{-3}$, which are usually found in the photosphere.

The peak of the microwave bulk emission was at about $9 \mathrm{GHz}$, with a spectral index of $\alpha=-2.3$ for higher frequencies (Gallagher et al. 2000). The value of maximum flux density at $15 \mathrm{GHz}$ was $1100 \mathrm{sfu}$ (Sol.-Geophys. Data 2000). Thus, the predicted fluxes for the bulk emission at $212 \mathrm{GHz}$ ( $2.5 \mathrm{sfu})$ and at $405 \mathrm{GHz}(0.6 \mathrm{sfu})$ would not be detectable by SST. On the other hand, the brighter submillimeter-wave spikes with $\alpha \sim 2$ would produce a flux of about $1 \mathrm{sfu}$ at $15 \mathrm{GHz}$ and less at lower frequency microwaves, not detectable by patrol solar telescopes.

The production of numerous spikes on large active regions and of multiple fast subsecond time structures in solar bursts has been known to occur at millimeter and centimeter waves (Kaufmann et al. 1980, 1985; Zodi, Kaufmann, \& Zirin 1984; Raulin et al. 1998; Nakajima 2000). Subsecond pulsations in the optical range have been recently found in flares (Wang et al. 2000; Trottet et al. 2000) and in quiescent active regions (Phillips 2001). They may have connection with microflares, waves, or quakes, with a possible important role in the heating of the solar corona (Sturrock \& Uchida 1981; Lin et al. 1984; Sturrock et al. 1984; Zharkova \& Kosovichev 2000). The physical mechanisms to explain the brighter discrete spikes at submillimeter waves have to account for the short timescales $(100-300 \mathrm{~ms})$ and a positive flux spectral index of about 2, raising difficult constraints for both thermal and nonthermal interpretations (Beckman 1968; Ohki \& Hudson 1975; Kaufmann et al. 1986; McClements \& Brown 1986; Klein 1987).

The SST efficiencies and beams have been optimized recently (2000 October), improving substantially the sensitivity and spatial resolution. Of particular interest will be the detailed study of the submillimeter-wave activity in space and time in comparison to high cadence rate $\mathrm{H} \alpha$ imaging of flaring sites (Wang et al. 2000; Trottet et al. 2000; Phillips 2001) and to time histories derived from images and light curves from the TRACE, SOHO, and HASTA instruments. High-energy counterparts will become available as the High-Energy Solar Spectroscopic Imager experiment is launched in 2001.

An anonymous referee gave useful suggestions improving the Letter's presentation. We acknowledge the technical assistance at the site by A. Marun and Y. R. Hadano and help in data reduction by A. M. Melo. Useful remarks were given by A. Magun. GOES X-rays were obtained from the NASA Goddard Space Flight Center Solar Data Analysis Center. This research was funded by the Brazilian research agency Fundação de Amparo a Pesquisa do Estado de São Paulo grant 99/01626-7, with partial support from the Argentinian agency Consejo Nacional de Investigaciones Científicas y Técnicas and the Switzerland National Research Council.

\section{REFERENCES}

Altenhoff, W. J., Baars, J., Downes, T., \& Wink, J. E. 1987, A\&A, 184, 381 Bagalá, L. G., Bauer, O. H., Fernández-Borda, R., Francile, C., Haerendel, G., Rieger, E., \& Rovira, M. 1999, in Proc. Ninth European Meeting on Solar Physics: Magnetic Fields and Solar Processes, ed. A. Wilson (ESA SP-449; Noordwijk: ESA), 469

Beckman, J. E. 1968, Nature, 220, 52

Clark, C. D., \& Park, W. M. 1968, Nature, 219, 922

Croom, D. L. 1973, in High Energy Phenomena on the Sun, ed. R. Ramaty \& R. G. Stone (NASA SP-342; Greenbelt: NASA/GSFC), 114

Gallagher, P. T., Gary, D. E., \& Lee, J. 2000, BAAS, 32, 821

Giménez de Castro, C. G., Raulin, J.-P., Makhmutov, V. S., Kaufmann, P., \& Costa, J. E. R. 1999, A\&AS, 140, 373

Hudson, H. S. 1975, Sol. Phys., 45, 69

Kaufmann, P. 1996, in AIP Conf. Ser. 374, High-Energy Solar Physics Workshop, ed. R. Ramaty, N. Mandzhavidze, \& X. M. Hua (New York: AIP), 379

Kaufmann, P., Correia, E., Costa, J. E. R., \& Zodi Vaz, A. M. 1986, A\&A, 157,11

Kaufmann, P., Correia, E., Costa, J. E. R., Zodi Vaz, A. M., \& Dennis, B. R. 1985, Nature, 313, 380

Kaufmann, P., Strauss, F. M., Raffaelli, J. C., \& Opher, R. 1980, in SolarTerrestrial Predictions Proc., Vol. 3, ed. R. F. Donnelly (Boulder: NOAA/ SEL), C115

Kaufmann, P., et al. 2000, in ASP Conf. Ser. 206, High Energy Solar Physics: Anticipating HESSI, ed. R. Ramaty \& N. Mandzhavidze (San Francisco: ASP), 318

Klein, K.-L. 1987, A\&A, 183, 341

Lin, R. P., Schwartz, R. A., Kane, S. R., Pelling, R. M., \& Hurley, K. C. 1984, ApJ, 283, 421
Makhmutov, V. S., Costa, J. E. R., Raulin, J.-P., Kaufmann, P., Lagrotta, P. R., Giménez de Castro, C. G., Magun, A., \& Arzner, K. 1998, Sol. Phys., 178,393

McClements, K. G., \& Brown, J. C. 1986, A\&A, 165, 235

Nakajima, H. 2000, in ASP Conf. Ser. 206, High Energy Solar Physics: Anticipating HESSI, ed. R. Ramaty \& N. Mandzhavidze (San Francisco: ASP), 313

Ohki, K., \& Hudson, H. S. 1975, Sol. Phys., 43, 405

Phillips, K. J. H. 2001, in IAU Symp. 203, Recent Insights into the Physics of the Sun and the Heliosphere-Highlights from $\mathrm{SOHO}$ and Other Space Missions, ed. P. Brekke, B. Fleck, \& J. B. Gurman (San Francisco: ASP), in press

Raulin, J.-P., Kaufmann, P., Olivieri, R., Correia, E., Makhmutov, V. S., \& Magun, A. 1998, ApJ, 498, L173

Shklovsky, J. 1964, Nature, 202, 275

Sol.-Geophys. Data. 2000, 668(1) (Boulder: NOAA)

Stein, W. A., \& Ney, E. P. 1963, J. Geophys. Res., 68, 65

Sturrock, P. A., Kaufmann, P., Moore, R. L., \& Smith, D. F. 1984, Sol. Phys., 94, 341

Sturrock, P. A., \& Uchida, Y. 1981, ApJ, 246, 331

Trottet, G., Rolli, E., Magun, A., Barat, C., Kuznetsov, A., Sunyaev, R., \& Terekhov, O. 2000, A\&A, 356, 1067

Wang, H., Qiu, J., Denker, C., Spirock, T. J., Chen, H., \& Goode, P. R. 2000, ApJ, 542, 1080

Zharkova, V. V., \& Kosovichev, A. G. 2000, in ASP Conf. Ser. 206, High Energy Solar Physics: Anticipating HESSI, ed. R. Ramaty \& N. Mandzhavidze (San Francisco: ASP), 77

Zodi, A. M., Kaufmann, P., \& Zirin, H. 1984, Sol. Phys., 92, 283 